\title{
Positive Affect through Interactions in Meetings: The Role of Proactive and Supportive Statements
}

\author{
Kurt Schneider, Jil Klünder, Fabian Kortum, Lisa Handke,2 Julia Straube, \\ Simone Kauffeld ${ }^{2}$
}

\begin{abstract}
Meetings often dominate software projects. Despite frequent dissatisfaction within meetings, many software engineers are not aware of the crucial importance about their behavior. This can set the tone for the entire project and influence the success. In a study based on 32 student development teams with 155 participants, we observed the participants' interactions during the first internal team meeting. Analyzing the observations showed that constructive remarks had a positive impact on positive group affect tone. However, this effect can only be observed when supportive utterances followed constructive remarks. In the article, we show a complete mediation of this statistically significant effect, e.g., about seemingly subtle interaction patterns that influence group affect tone. We also propose practical interventions on how software projects could benefit from supportive meeting behavior. This summary refers to the article "Positive affect through interactions in meetings: The role of proactive and supportive statements" [Sc18] published in the Journal of Systems \& Software in 2018 (vol. 143).
\end{abstract}

Keywords: Positive group affect tone (PGAT), proactive and supportive statements, empirical study

\section{Introduction}

Most software projects require some sort of team work. In order to ensure an appropriate information exchange and the synchronization of all team members, meetings are part of most projects. The success of a meeting in the means of satisfied participants and desired meeting outcomes strongly depends on the interactions during the meetings. While proactive and structuring statements can support meeting success, destructive behavior endangers meeting success - and influences the further project. However, a lot of meeting participants are not aware of the relevance of their behavior. Interaction analysis can help to reveal appropriate and inappropriate behavior in meetings and to increase the participants' awareness of the relevance of their behavior [KLW12]. In particular, appropriate behavior such as proactive statements in meetings can support positive affect afterwards.

\footnotetext{
${ }^{1}$ Software Engineering Group, Leibniz Universität Hannover, Welfengarten 1, 30167 Hannover, Germany \{kurt.schneider,jil.kluender,fabian.kortum\}@inf.uni-hannover.de

${ }^{2}$ Department of Industrial, Organizational and Social Psychology, Technische Universität Braunschweig, Spielmannstr. 19, 38106 Braunschweig, Germany \{1.handke,julia.straube,s.kauffeld $\} @$ tu-braunschweig.de
} 


\section{Study and Results}

In a large-scale study, we analyzed the first team meeting of 32 student development teams. Each team consisted of 3 to 5 students, leading to a total number of 155 participants. Each of the meetings was analyzed using the established act4teams coding scheme [KLW12]. Applying this coding scheme leads to a fine-grained overview of the number of different types of statements, such as supporting, interrupting or complaining statements. In our study, we concentrated on supportive and proactive statements. Supportive statements are given by agreements to suggestions or ideas, e.g., "yes, this makes sense...". Proactive statements are mainly given by signalizing interest in change, e.g., "Let's assume we are successful...". Correlation analysis comparing the number of different statements with self-reported data on positive and negative affect leads to three core statements [Sc18]:

- The rate of proactive statements within a meeting does not significantly influence positive group affect after the meeting.

- $\quad$ Positive group affective tone is triggered by proactive statements via support.

- The probability of a supportive statement is significantly higher when a proactive statement has been made.

\section{Conclusion}

Our results show the importance of appropriate behavior in meetings and its influence on the success of a meeting. In particular, our results show that "a verbal and content-oriented utterance has a measurable impact on positive affect" [Sc18]. Consequently, not only direct and non-verbal stimuli influences positive affect, but also serious constructive statements. Furthermore, positive affect is supported by triggers and support from other meeting participants.

\section{Literaturverzeichnis}

[KLW12] Kauffeld, Simone; Lehmann-Willenbrock, Nale: Meetings matter: Effects of team meetings on team and organizational success. Small Group Research, 43(2):130-158, 2012.

[Sc18] Schneider, Kurt; Klünder, Jil; Kortum, Fabian; Handke, Lisa; Straube, Julia; Kauffeld, Simone: Positive affect through interactions in meetings: The role of proactive and supportive statements. Journal of Systems and Software, 143:59-70, 2018. 